\title{
ON GENERATING HEAVY POINTS WITH POSITIVE MATRICES
}

\author{
A. K. SNYDER ${ }^{1}$
}

Let $S$ be a countable topological space. If $S$ is indexed by the positive in tegers, then real functions on $S$ may be considered as sequences and are subject to infinite matrix transformation. After Henriksen and Isbell in [3], a point $s$ in $S$ is called a heavy point if there exists a regular matrix which sums every bounded real-valued function on $S$ continuous at $s$. It is clear that the notion of a heavy point is independent of the order chosen for $S$. Some properties of heavy points are given in [3]. For example, sequential limit points are heavy points and isolated points (in fact, points whose complements are $C^{*}$ embedded) are not heavy points. In addition Henriksen and Isbell observe that not all heavy points are sequential limit points. The latter phenomenon was the motivation for the present paper.

Corresponding to each positive regular matrix $A$ we define a topology on the positive integers by taking neighborhoods of 1 to be sets whose characteristic functions are $A$-summable to 1 . Denote the resulting space by $N_{A}$. The point 1 is a heavy point in $N_{A}$. Some basic properties of these spaces are examined. Also, we determine the class of matrices $A$ for which 1 is not a sequential limit point in $N_{A}$.

Let $A=\left(a_{n k}\right)$ be an infinite real matrix. The $A$-transform of the real sequence $x=\left\{x_{k}\right\}$ is the sequence $\left\{\sum_{k} a_{n k} x_{k}\right\}$ if these sums exist. Let $c$ and $m$ denote the Banach spaces of convergent sequences and bounded sequences, respectively, with $\|x\|=\sup _{k}\left|x_{k}\right|$. Let $c_{A}$ $=\{x: A x \in c\}$. The matrix $A$ is said to sum the members of $c_{A}$ or any subset of $c_{A}$. Define the functional $\lim _{A}$ on $c_{A}$ by $\lim _{A} x=\lim A x$. The matrix $A$ is called conservative if $c \subset c_{A}$ and regular if in addition $\lim =\lim _{A}$ on $c$. It is well known that $A$ is regular if and only if $\lim _{n} a_{n k}=0$ for all $k, \lim _{n} \sum_{k} a_{n k}=1$, and $\sup _{n} \sum_{k}\left|a_{n k}\right|<\infty$. The matrix $A$ is called positive if $a_{n k} \geqq 0$ for all $n, k$.

For a set $T$ of positive integers let $\chi(T)$ denote the characteristic function of $T ;(\chi(T))_{k}=1$ for $k \in T, 0$ otherwise.

For a topological space $S$ let $C^{*}(S)$ denote the Banach space of bounded real-valued continuous functions on $S$ with $\|x\|$ $=\sup \{|x(s)|: s \in S\}$. As observed in [3], if $S$ is countable and completely regular, then $S$ is 0 -dimensional (i.e. the ring $\Gamma$ of open and

Received by the editors December 8, 1965.

1 This work is part of the author's doctoral dissertation written at Lehigh University under the direction of Professor A. Wilansky. 
closed sets is a base for the topology of $S$ ) and the linear span of $\{\chi(U): U \in \Gamma\}$ is dense in $C^{*}(S)$.

Note that a conservative matrix $A$ may be considered as a bounded linear transformation on $m$ and that $c$ is closed in $m$. It follows that $c_{A} \cap m$ is closed in $m$. Hence, if $A$ sums $\{\chi(U): U \in \Gamma\}$, then it sums $C^{*}(S)$.

Let $\mathcal{F}$ denote the set of finite subsets of $N \sim\{1\}$ where $N$ is the positive integer. Let $R$ be a ring of subsets of $N$ which includes $\mathcal{F}$ and $N$. Let $\mu$ be a real finitely additive function on $R, \mu \geqq 0, \mu(N)=1$, and $\mu=0$ on $\mathfrak{F}$. Finally, let $B_{\mu}=\{U \cup\{1\}: U \in R, \mu(U)=1\}$. Then $B_{\mu} \cup \mathfrak{F}$ is a base for a topology on $N$. The resulting space, call it $N_{\mu}$, is Hausdorff, completely regular, and has at most one limit point. Actually, any countable space with these properties may be obtained as an $N_{\mu}$ space.

We shall be concerned here with the case in which $\mu$ is given by a positive regular matrix $A$. Let $R=\left\{U: \lim _{A} \chi(U)=1\right.$ or 0$\}, \mu(U)$ $=\lim _{A} \chi(U)$. Denote the resulting space by $N_{A}$.

Examples. If $A$ is the identity matrix, then $n \rightarrow 1$ in $N_{A}$.

Let $B$ be the $(C, 1)$ matrix, $b_{n k}=1 / n$ for $k \leqq n, 0$ otherwise. Then $N_{B}$ is the space given by Appert in [2, p. 84]. Neighborhoods of the point 1 are sets with density 1 . As pointed out by Sierpinski [4, p. 61] the space $N_{B}$ is not first countable.

Let $D$ be the matrix defined by $d_{n k}=2^{-p}$ for $k=2^{p-1}(2 n-1), 0$ otherwise. Then $N_{D}$ is the space given in $[4$, p. 61]. Here 1 is a sequential limit point, but $N_{D}$ is not first countable.

Corresponding to $A=\left(a_{n k}\right)$, let $a_{A}^{\boldsymbol{k}}=\sup _{n} a_{n k}$.

Lemma. Let $S=\left\{n_{k}\right\}$ be an infinite subset of $N_{A} \sim\{1\}$.

(i) If $\lim _{A} \chi(S)=0$, then $a_{A}^{n} \rightarrow 0$.

(ii) $S$ includes an infinite closed subset of $N_{A}$ if and only if $\lim _{\inf } a_{A}^{n_{k}}$ $=0$.

Proof. The proof of (i) is easy and is omitted.

Assume $\lim \inf _{k} a_{A}^{n_{k}}=0$. Choose distinct positive integers $r_{k}$ such that $\sum_{k} a_{A}^{n_{r}}<\infty$. Let $T=\left\{n_{r_{k}}: k=1,2, \cdots\right\}$. Then $\lim _{A} \chi(T)$ $=\lim _{n} \sum_{k} a_{n, n_{r_{k}}}=0$, since the series is uniformly convergent. Hence, $T$ is closed in $N_{A}$ by definition. The converse follows from (i).

Theorem. (i) $N_{\mu}$ is pseudo-finite (i.e. all compact sets are finite) if and only if 1 is not a sequential limit point.

(ii) A sums $C^{*}\left(N_{A}\right)$; hence, 1 is a heavy point in $N_{A}$.

(iii) $N_{A}$ is not extremally disconnected.

(iv) 1 fails to be a sequential limit point in $N_{A}$ if and only if $a_{A}^{k} \rightarrow 0$.

(v) $N_{A}$ is first countable if and only if it is locally compact. 
Proof. Part (i) is immediate.

It is clear that $A$ sums $\chi(U)$ for every open and closed subset $U$ of $N_{A}$, so part (ii) follows.

Actually, (iii) follows from (ii) and Theorem 3 of [3]. In our setting it is easier to choose a subset $S$ of $N_{A} \sim\{1\}$ such that $\chi(S) \notin c_{A}$. (See [5, p. 54].) Then $S$ and $T=N_{A} \sim(S \cup\{1\})$ are disjoint open sets, but $1 \in \bar{S} \cap \bar{T}$. Hence, $N_{A}$ is not extremally disconnected.

Part (iv) follows from part (ii) of the lemma and the fact that 1 is a sequential limit point if and only if there exists an infinite subset of $N_{A} \sim\{1\}$ which includes no infinite closed subset of $N_{A}$.

If $N_{A}$ is locally compact, then it is first countable by $[1$, p. 65 , Theorem III], since every point of $N_{A}$ is a $G_{\delta}$. Conversely, let $\left\{U_{k}\right\}$ be a strictly decreasing basic sequence of neighborhoods of 1 in $N_{A}$. Now $\lim _{A} \chi\left(U_{k} \sim U_{k+1}\right)=0$ for each $k$. If the set $U_{k} \sim U_{k+1}$ is frequently infinite, then using (i) of the lemma we may choose $n_{k} \in U_{k}$ $\sim U_{k+1}$ for each $k$ such that $\lim \inf _{k} a_{A}^{n} k=0$. By (ii) of the lemma, there exists a closed subset of $N_{A} \sim\{1\}$ which meets every $U_{k}$, contradicting the fact that $\left\{U_{k}\right\}$ is basic. If $U_{k} \sim U_{k+1}$ is finite, say for $k \geqq p$, then $U_{p}$ is a compact neighborhood of 1 and $N_{A}$ is locally compact. This completes the proof.

Note that half of part (v) fails for $N_{\mu}$ spaces in general.

It is clear that the compact $N_{A}$ spaces are identical. Likewise, noncompact but locally compact $N_{A}$ spaces are homeomorphic. However, $N_{A}$ spaces which are not locally compact need not be homeomorphic. In fact we have

ExAmple. Let $A$ be the $(C, 1)$ matrix. Let $B=\left(b_{n k}\right)$ be defined by $b_{n k}=1 / n$ for $n(n-1) / 2<k \leqq n(n+1) / 2,0$ otherwise. According to (iv) of the theorem, 1 is not a sequential limit point of $N_{A}$ or $N_{B}$. However, corresponding to each permutation $\pi$ of $N$ there exists a set $S$ in $N$ with $\lim _{B} \chi(S) \neq 1$ but which has density 1 with respect to $\pi$. Hence, $N_{A}$ is not homeomorphic to $N_{B}$.

\section{REFERENCES}

1. P. Alexandroff and P. Urysohn, Memoire sur les espaces topologiques compacts, Verh. Nederl. Akad. Wetensch. Afd. Natuurk. Sect. I 14 (1929), 1-93.

2. A. Appert, Proprietés des espaces abstraits les plus généraux, Actualités Sci. Indust. No. 146, Hermann, Paris, 1934.

3. M. Henriksen and J. R. Isbell, Averages of continuous functions on countable spaces, Bull. Amer. Math. Soc. 70 (1964), 287-290.

4. W. Sierpinski, General topology, Univ. of Toronto Press, Toronto, 1956.

5. K. Zeller, Theorie der Limitierungsverfahren, Springer-Verlag, Berlin, 1958.

Massachusetts Institute of Technology 